\title{
Physical activity participation is associated with higher quality of life scores in men with alcohol use disorders: a study from Uganda
}

\author{
Davy Vancampfort ${ }^{1,2}$, Mats Hallgren ${ }^{3}$, Byamah Brian Mutamba ${ }^{4}$, Tine Van Damme ${ }^{1}$, \\ Michel Probst ${ }^{1}$, Ruud van Winkel, ${ }^{2,5}$, Inez Myin-Germeys ${ }^{5}$, Marc De Hert ${ }^{2}$, James Mugisha ${ }^{4,6}$
}

1. KU Leuven Department of Rehabilitation Sciences, Leuven, Belgium

2. KU Leuven, University Psychiatric Center KU Leuven, Leuven-Kortenberg, Belgium

3. Department of Public Health Sciences, Karolinska Institutet, Stockholm, Sweden

4. Butabika National Referral Mental Hospital, Kampala, Uganda

5. KU Leuven, Centre for Contexual Psychiatry, Leuven, Belgium

6. Kyambogo University, Kampala, Uganda

\begin{abstract}
Background: There is a growing recognition of the importance of encouraging people with alcohol use disorders (AUD) to become more active as an achievable strategy to reduce the disability-associated burden.

Objective: We investigated whether physical activity and sedentary behaviour in men with AUD contribute to their quality of life (QoL).

Methods: Fifty male Ugandan inpatients with AUD (33.0土10.7 years) completed the World Health Organization Quality of Life Assessment brief version, Simple Physical Activity Questionnaire and the Alcohol Use Disorders Identification Test while waist circumference, body mass index and blood pressure were assessed. Linear multiple regression analysis explored the total variance in QoL explained by all predictor variables.

Results: SIMPAQ walking and SIMPAQ exercise explained $46 \%$ of the variability in physical QoL, $45 \%$ of the variability in psychological QoL, and $40 \%$ of the variability in environmental QoL. The SIMPAQ walking score predicted 37\% of the variability in social QoL.

Conclusion: The current findings suggest that higher levels of walking and exercising are associated with a better QoL. Our study therefore provides a platform for future research to investigate the role of physical activity on QoL levels in people with AUD, also in low resourced settings in low-income countries such as Uganda.
\end{abstract}

Keywords: Physical activity; exercise; alcohol; quality of life.

DOI: https://dx.doi.org/10.4314/ahs.v20i3.46

Cite as: Vancampfort D, Hallgren M, Mutamba BB, Van Damme T, Probst M, van Winkel R, et al. Physical activity participation is associated with higher quality of life scores in men with alcohol use disorders: a study from Uganda. Afri Health Sci. 2020;20(3): 1407-1415. bttps:/ / dx.doi.org/10.4314/abs.v20i3.46

\section{Introduction}

Alcohol use disorders (AUD) have an estimated global lifetime prevalence of around $16 \%{ }^{1}$. Worldwide more than $5 \%$ of the global burden of disease is attributable to alcohol consumption ${ }^{2}$, and harmful alcohol use
Corresponding author:
Davy Vancampfort,
Tervuursevest 1013001 Leuven,
Belgium
Tel.: +32 275805 11; Fax: +32 27599879 .
E-mail address: davy.vancampfort@kuleuven.be

causes almost 3.5 million deaths every year (about 6\% of all deaths). Compared with the general population, people with AUD have a 10-fold risk of mortality from liver cirrhosis, a seven-fold risk for injury fatalities and a two-fold risk for cardiovascular and cancer deaths ${ }^{3}$. The current treatment of AUD includes pharmacotherapy, (cognitive) behavioural therapy and mutual peer support programs ${ }^{1}$. Despite advancements in these treatment modalities, relapse remains high while pharmacotherapy has unwanted side-effects and compliance is often low ${ }^{4}$. 
It could be hypothesized that the chronic nature of AUD including the recurrence of risky drinking patterns, side-effects of pharmacotherapy and the high prevalence of somatic co-morbidities might have a major impact on the physical and psychological domains of quality of life (QoL ${ }^{5,6}$. QoL is an important patient-rated outcome that measures the impact a disorder, such as AUD has on a person's general well-being and life satisfaction. It is also a relevant outcome for policy makers, researchers and clinicians. The importance of considering QoL in AUD treatment research has however only recently been voiced ${ }^{7}$. Previous research has demontrated that the health-related QoL of moderate alcohol drinkers is higher than that of heavy drinkers, as defined with the Alcohol Use Disorders Identification Test (AUDIT) ${ }^{8,9}$, while disability due to chronic conditions is negatively associated with perceived QoL in people with AUD ${ }^{6}$.

There is a growing recognition of the importance of encouraging patients with AUD to become more active as an achievable strategy to reduce this disability-associated burden and improve QoL ${ }^{10-13}$. Without an understanding of the current QoL status and associations with current physical activity levels, it is however difficult to advise people with AUD how to optimize their physical activity habits to improve their QoL. Next to this, it is unknown whether sedentary behavior is also associated with QoL in those with AUD. Sedentary behavior can be defined as an energy expenditure $\leq 1.5$ metabolic equivalents of task, while in a sitting or reclining posture during waking hours ${ }^{14}$. It is associated with increased cardio-metabolic risks and premature mortality independent from physical activity levels ${ }^{15}$. The specific aim of the present study was to investigate whether physical activity and sedentary levels in people with AUD contribute to a person's QoL, while adjusting for previously established contributing factors such as age, the presence of cardio-metabolic risks and the level of alcohol consumption.

\section{Methods}

\section{Design}

This is a cross-sectional study.

\section{Participants and procedure}

During a 3-month period, all consecutive male inpatients of the alcohol unit of the Butabika National Referral Mental Hospital, Kampala, Uganda who had a clinical DSM 5 diagnosis ${ }^{16}$ of AUD as diagnosed by the treating psychiatrist were invited to participate. Only men were included as female patients were not treated at the time of testing in the inpatient alcohol unit. Also only inpatients who were treated at least for 4 weeks on the ward and for whom medication was stable were invited. Although detoxification and psychotherapy are the basis of the treatment protocol, inpatients do have the option to exercise once a day (walking, soccer, basketball, netball). All questionnaires were interviewer-administered in English or Luganda when appropriate. The study procedure was approved by the ethical committee of Mengo Hospital and the Butabika Hospital Research Committee (Ref. No. = 997/3-17). All participants gave their written informed consent. Data were analysed anonymously.

\section{World Health Organization Quality of Life Assess- ment brief version (WHOQoL-BREF)}

Quality of life during the previous 4 weeks was assessed using the WHOQo-BREF ${ }^{17}$. The WHOQoL-BREF is a 26-item questionnaire developed from the original 100item questionnaire; the WHOQoL-100. The WHOQoL-BREF covers four different subscales: physical health (energy and fatigue, pain and discomfort, sleep and rest), psychological health (body image and appearance, negative feelings, positive feelings, self-esteem, thinking, learning, memory and concentration), social relationships (personal relationships, social support and sexual activity) and environment (e.g. financial resources, freedom, physical safety and security, health and social care). Each individual item of the WHOQoL-BREF is scored from one to five on a response scale. The scores are then transformed linearly to a 0-100 scale. Higher scores indicate a better QoL.

\section{Simple Physical Activity Questionnaire}

The SIMPAQ (http://www.simpaq.org) ${ }^{18}$ is a 5 -item clinical tool to assess physical activity among populations at high risk for sedentary behavior. The SIMPAQ accounts for time spent in bed overnight (box 1), time sedentary, including napping (box 2), time spent walking (box 3), time spent exercising (box 4) and time engaged in incidental activity (box 5), averaged over the past seven-day period. The sum of the hours recorded in the five SIMPAQ boxes should add to approximately 24-hours, providing interviewers with an opportunity to clarify with participants if significant under or over-reporting has occurred (e.g. total of $<18$ hours or $>30$ hours accounted for). In this study, we used the time spent sedentary during waking hours (hours/day), 
time spent walking ( $\mathrm{min} /$ day), time spent in structured exercise ( $\mathrm{min} /$ day), and time spent in incidental or non-structured physical activity (min/day) during the past week.The SIMPAQ showed an excellent reliability in Ugandan patients with mental illness (data submitted).

\section{Alcohol Use Disorders Identification Test (AU- DIT)}

To assess risky alcohol consumption patterns in this study, we used the AUDIT ${ }^{19}$, which was developed by the World Health Organization (WHO) as a simple method of screening for excessive drinking. The AUDIT comprises three domains: hazardous alcohol use (frequency of drinking, typical quantity, and frequency of heavy drinking), dependence symptoms (impaired control over drinking, increased salience of drinking, and morning drinking), and harmful alcohol use (guilt after drinking, blackouts, alcohol-related injuries, and other concerns about drinking). Higher scores indicate greater likelihood of hazardous and harmful drinking. We used the AUDIT as a continuous score.

\section{Metabolic assessments}

Waist circumference (WC) was measured to nearest $1 \mathrm{~cm}$ at the level of the umbilicus and at the end of expiration with the subject upright. Blood pressure measurements were taken on the left arm with the participant in the sitting position using a calibrated electronic blood pressure device. For calculating the body mass index (BMI), body weight was measured in light clothing to the nearest $0.1 \mathrm{~kg}$ using a SECA beam balance scale, and height to the nearest $0.1 \mathrm{~cm}$ using a wall-mounted stadiometer.

\section{Statistical analyses}

Data were assessed for normality using the Shapiro-Wilk test and SIMPAQ data was found not to be normally distributed. However, in order to allow comparison with other physical activity studies, descriptive statistics are presented as mean and standard deviation (SD). As a first step, Pearson correlation or Spearman Rho coefficients were calculated when appropriate to estimate the strength of association between physical, psychological, social and environmental QoL, and single variables of interest. Differences in WHOQoL-BREF scores between the risk groups were assessed with independent samples t-tests. All significant correlates were included in a linear multiple regression analysis in order to assess the total variance in QoL explained by all predictor variables. To assess whether the associations with QoL outcomes differed by age and BMI, the terms representing interactions between each clinical measure and age and BMI were added to the main effects regression equation. The significance level was set at $\mathrm{P}<0.05$. SPSS 24.0 was used for the data analyses (SPSS Inc, Chicago, IL).

\section{Results \\ Participants}

A total of 54 consecutive inpatients with AUD were invited to take part in the study. Two patients were excluded due to somatic contra-indications, which would prevent them from being physically active as usual, and another two patients declined to participate. None of the patients who agreed to participate dropped out and there were no missing data. In total, 50 men were included. The mean age was $33.0 \pm 10.7$ years, mean height $170.0 \pm 8.0 \mathrm{~cm}$, mean BMI $21.8 \pm 2.9$, mean waist circumference $81.8 \pm 10.0 \mathrm{~cm}$, and mean systolic and diastolic blood pressure $113.5 \pm 13.8 \mathrm{mmHg}$ and $73.2 \pm 9.6$ $\mathrm{mmHg}$, respectively. Sixteen patients $(32 \%)$ smoked a mean of $1.2 \pm 0.6$ cigarettes per day. The mean and SD of the SIMPAQ, AUDIT and WHOQoL-BREF scores are presented in Table 1. 
Table 1. Means and standard deviations of socio-demographic, clinical, physical activity and sedentary behavior levels and Pearson or Spearman Rho correlations with WHOQoL-BREF scores in men with alcohol use disorders $(\mathrm{n}=50)$

\begin{tabular}{|c|c|c|c|c|c|c|c|c|c|}
\hline & \multirow[t]{2}{*}{ Mean \pm SD } & \multicolumn{2}{|c|}{$\begin{array}{c}\text { Physical } \\
(68.0 \pm 14.3)\end{array}$} & \multicolumn{2}{|c|}{$\begin{array}{c}\text { Psychological } \\
(64.7 \pm 15.8)\end{array}$} & \multicolumn{2}{|c|}{$\begin{array}{c}\text { Social } \\
(55.9 \pm 18.7)\end{array}$} & \multicolumn{2}{|c|}{$\begin{array}{c}\text { Evironmental } \\
(56.4 \pm 11.9)\end{array}$} \\
\hline & & $r$ & $P$ & $\bar{R}$ & $P$ & $r$ & $P$ & $r$ & $P$ \\
\hline Age (years) & $33.0 \pm 10.7$ & 0.22 & 0.13 & 0.14 & 0.34 & 0.14 & 0.34 & 0.10 & 0.48 \\
\hline $\begin{array}{l}\text { Body mass } \\
\text { index }\left(\mathrm{kg} / \mathrm{m}^{2}\right)\end{array}$ & $21.8 \pm 2.9$ & 0.09 & 0.55 & -0.03 & 0.83 & -0.10 & 0.50 & 0.16 & 0.27 \\
\hline $\begin{array}{l}\text { Waist } \\
\text { circumference } \\
(\mathrm{cm})\end{array}$ & $81.8 \pm 10.0$ & -0.05 & 0.71 & -0.11 & 0.46 & -0.23 & 0.11 & 0.22 & 0.13 \\
\hline $\begin{array}{l}\text { Smoking } \\
(n=16)\end{array}$ & $1.2 \pm 0.6$ & 0.38 & 0.15 & -0.03 & 0.91 & 0.07 & 0.78 & 0.22 & 0.41 \\
\hline $\begin{array}{l}\text { SIMPAQ } \\
\text { walking } \\
\text { (min/day) }{ }^{\circ}\end{array}$ & $18.9 \pm 18.3$ & 0.41 & $0.003 *$ & 0.43 & $0.002 *$ & 0.30 & $0.036^{*}$ & 0.36 & $0.01 *$ \\
\hline $\begin{array}{l}\text { SIMPAQ } \\
\text { exercise } \\
(\min / \text { day })^{\circ}\end{array}$ & $4.6 \pm 9.0$ & 0.34 & $0.016^{*}$ & 0.37 & $0.008 *$ & 0.24 & 0.09 & 0.28 & $0.048 *$ \\
\hline $\begin{array}{l}\text { SIMPAQ } \\
\text { incidental } \\
\text { (min/day) }^{\circ}\end{array}$ & $18.3 \pm 39.9$ & 0.22 & 0.13 & -0.04 & 0.74 & -0.14 & 0.34 & -0.02 & 0.89 \\
\hline $\begin{array}{l}\text { SIMPAQ } \\
\text { sedentary } \\
\text { (hours/day) }^{\circ}\end{array}$ & $4.2 \pm 3.4$ & 0.04 & 0.77 & 0.02 & 0.91 & -0.19 & 0.18 & 0.06 & 0.70 \\
\hline $\begin{array}{l}\text { AUDIT (total } \\
\text { continuous } \\
\text { score) }\end{array}$ & $18.1 \pm 8.4$ & -0.12 & 0.41 & 0.01 & 0.92 & -0.16 & 0.26 & -0.004 & 0.97 \\
\hline
\end{tabular}

${ }^{\circ}$ Spearman Rho correlation; *Significant when $\mathrm{P}<0.005$; WHOQoL-BREF=World Health Organization Quality of Life Assessment brief version, SIMPAQ=Simple Physical Activity Questionnaire, AUDIT= Alcohol Use Disorders Identification Test.

\section{Correlates with WHOQoL-BREF scores}

Higher SIMPAQ walking levels were significantly $(\mathrm{P}<0.05)$ associated with better physical, psychological, social and environmental WHOQoL-BREF scores, while higher SIMPAQ exercise scores were significantly $(\mathrm{P}<0.05)$ associated with better physical, psychologi$\mathrm{cal}$, and environmental WHOQo-BREF scores. The rand $\mathrm{P}$-values are presented in Table 1. Age, BMI, waist circumference, SIMPAQ incidental levels and AUDIT scores were not significantly associated with any WHOQoL-BREF scores.

\section{Regression analyses of WHOQoL-BREF scores}

Following the correlation analyses, variables significantly correlated with the different QoL domains were included in the regression analyses. To assess wheth- er associations with the QoL domains differed by age or BMI, interactions with each included correlate were added to the main effects regression equation. Tables 2 to 5 show that there were no significant age or BMI interactions with the SIMPAQ walking and SIMPAQ exercise scores. SIMPAQ walking and SIMPAQ exercise significantly predicted the physical, psychological and environmental QoL domains independently, but when both variables were included in the same regression model, neither remained a significant predictor. The final model including the SIMPAQ walking and SIMPAQ exercise explained $46 \%$ of the variability in physical QoL (see Table 2), 45\% of the variability in psychological QoL (see Table 3), and 40\% of the variability in environmental QoL (see Table 5). The SIMPAQ walking score predicted $37 \%$ of the variability in social QoL (see Table 4). 
Table 2. Physical WHOQoL-BREF regressed on time spent walking and time spent exercising

\begin{tabular}{|c|c|c|c|c|c|c|}
\hline & $b$ & $S E$ & beta & $t$ & $P$ & $r^{2}$ \\
\hline SIMPAQ walking & & & & & & 0.42 \\
\hline Min/day & 0.27 & 0.11 & 0.35 & 2.53 & $0.015^{*}$ & \\
\hline Age (years) & 0.20 & 0.19 & 0.15 & 1.09 & 0.28 & \\
\hline BMI & 0.46 & 0.70 & 0.09 & 0.66 & 0.51 & \\
\hline Constant & 45.61 & 15.86 & & 2.88 & $0.006^{*}$ & \\
\hline SIMPAQ exercise (min/day) & & & & & & 0.42 \\
\hline Min/day & 0.57 & 0.22 & 0.22 & 2.54 & $0.015^{*}$ & \\
\hline Age (years) & 0.30 & 0.18 & 0.18 & 1.61 & 0.11 & \\
\hline BMI & 0.46 & 0.70 & 0.09 & 0.66 & 0.51 & \\
\hline Constant & 45.38 & 15.87 & & 2.86 & $0.006^{*}$ & \\
\hline All together & & & & & & 0.46 \\
\hline $\begin{array}{l}\text { SIMPAQ walking } \\
\text { (min/day) }\end{array}$ & 0.18 & 0.12 & 0.23 & 2.76 & 0.16 & \\
\hline $\begin{array}{l}\text { SIMPAQ exercise } \\
\text { (min/day) }\end{array}$ & 0.38 & 0.26 & 0.23 & 1.44 & 015 & \\
\hline Age (years) & 0.24 & 0.19 & 0.18 & 1.45 & 0.21 & \\
\hline BMI & 0.52 & 0.69 & 0.10 & 1.27 & 0.46 & \\
\hline Constant & 43.49 & 15.73 & & 2.76 & $0.008^{*}$ & \\
\hline
\end{tabular}

Table 3. Pychological WHOQoL-BREF regressed on time spent walking and time spent exercising

\begin{tabular}{|c|c|c|c|c|c|c|}
\hline & $\mathbf{b}$ & SE & Beta & $\mathbf{t}$ & $\mathbf{P}$ & $\mathbf{r}^{2}$ \\
\hline SIMPAQ walking & & & & & & 0.40 \\
\hline Min/day & 0.32 & 0.12 & 0.38 & 2.67 & $0.011 *$ & \\
\hline Age (years) & 0.12 & 0.21 & 0.08 & 0.56 & 0.57 & \\
\hline BMI & -0.09 & 078 & -0.01 & -0.11 & 0.91 & \\
\hline Constant & 56.30 & 17.72 & & 3.18 & $0.003 *$ & \\
\hline SIMPAQ exercise (min/day) & & & & & & 0.40 \\
\hline Min/day & 0.68 & 0.25 & 0.38 & 2.71 & $0.010^{*}$ & \\
\hline Age (years) & 0.23 & 0.21 & 0.15 & 1.10 & 0.28 & \\
\hline BMI & -0.09 & 0.78 & -0.01 & -0.11 & 0.91 & \\
\hline Constant & 55.95 & 17.70 & & 3.16 & $0.003 *$ & \\
\hline All together & & & & & & 0.45 \\
\hline $\begin{array}{l}\text { SIMPAQ walking } \\
\text { (min/day) }\end{array}$ & 0.21 & 0.14 & 0.24 & 1.51 & 0.14 & \\
\hline $\begin{array}{l}\text { SIMPAQ exercise } \\
\text { (min/day) }\end{array}$ & 0.45 & 0.29 & 0.25 & 1.57 & 0.12 & \\
\hline Age (years) & 0.16 & 0.21 & 0.11 & 0.76 & 0.45 & \\
\hline BMI & -0.02 & 0.77 & -0.004 & -0.03 & 0.98 & \\
\hline Constant & 53.75 & 17.51 & & 3.07 & $0.004 *$ & \\
\hline
\end{tabular}


Table 4. Social WHOQoL-BREF regressed on time spent walking

\begin{tabular}{lcccccc}
\hline & b & SE & Beta & $\mathbf{t}$ & $\mathbf{P}$ & $\mathbf{r}^{\mathbf{2}}$ \\
\hline SIMPAQ walking & & & & & & 0.37 \\
Min/day & 0.32 & 0.14 & 0.33 & 2.29 & $0.027^{*}$ & \\
Age (years) & 0.19 & 0.25 & 0.11 & 0.77 & 0.45 & \\
BMI & -0.60 & 0.92 & -0.09 & -0.65 & 0.52 & \\
Constant & 55.93 & 20.95 & & 2.67 & $0.011^{*}$ & \\
\hline
\end{tabular}

$\mathrm{b}=$ unstandardised regression coefficient, beta $=$ standardised regression coefficient, $\mathrm{SE}=$ standard error, WHOQoL-BREF=World Health Organization Quality of Life Assessment brief version, SIMPAQ=Simple Physical Activity Questionnaire.

Table 5. Environmental WHOQoL-BREF regressed on time spent walking and time spent exercising

\begin{tabular}{lcccccc}
\hline & $\mathbf{b}$ & $\mathbf{S E}$ & beta & $\mathbf{t}$ & $\mathbf{P}$ & $\mathbf{r}^{\mathbf{2}}$ \\
\hline SIMPAQ walking & & & & & & 0.38 \\
$\quad$ Min/day & 0.22 & 0.09 & 0.34 & 2.36 & $0.023^{*}$ & \\
$\quad$ Age (years) & 0.03 & 0.16 & 0.03 & 0.18 & 0.85 & \\
$\quad$ BMI & 0.78 & 0.60 & 0.18 & 1.30 & 0.20 & \\
$\quad$ Constant & 34.25 & 13.54 & & 2.53 & $0.015^{*}$ & \\
SIMPAQ exercise (min/day) & & & & & & 0.34 \\
$\quad$ Min/day & 0.39 & 0.19 & 0.29 & 2.01 & $0.049^{*}$ & \\
$\quad$ Age (years) & 0.10 & 0.16 & 0.09 & 0.63 & 0.53 & \\
$\quad$ BMI & 0.76 & 0.61 & 0.18 & 1.25 & 0.22 & \\
$\quad$ Constant & 34.75 & 13.76 & & 2.52 & $0.015^{*}$ & \\
All together & & & & & & 0.40 \\
$\quad$ SIMPAQ walking & 0.16 & 0.11 & 0.25 & 1.52 & 0.14 & \\
(min/day) & & & & & & \\
$\quad$ SIMPAQ exercise & 0.22 & 0.22 & 0.16 & 0.96 & 0.34 & \\
(min/day) & & & & & & \\
$\quad$ Age (years) & 0.05 & 0.16 & 0.04 & 0.30 & 0.77 & \\
$\quad$ BMI & 0.81 & 0.60 & 0.19 & 1.35 & 0.18 & \\
$\quad$ Constant & 33.03 & 13.61 & & 2.43 & $0.019^{*}$ & \\
\hline
\end{tabular}

$\mathrm{b}=$ unstandardised regression coefficient, beta $=$ standardised regression coefficient, $\mathrm{SE}=$ =standard error, WHOQ $\mathrm{oL}-$ BREF=World Health Organization Quality of Life Assessment brief version, SIMPAQ=Simple Physical Activity Questionnaire.

\section{Discussion}

\section{General findings}

To the best of our knowledge, the current study is the first to investigate associations between physical activity, sedentary behaviour and QoL in people with AUD. Our data demonstrate that higher levels of walking and structured exercise were, independent from known risk factors for low QoL such as older age, metabolic risks and high-risk drinking patterns, significantly associated with a higher QoL. Importantly, results show that walking and exercise are associated with better physical, psychological, and environmental QoL while walking was only associated with a better social QoL. Sedentary behavior, on its turn, was not associated with any WHOQoL-BREF scores.
Our data therefore indicate that people who walk and exercise more, experience higher energy levels, less fatigue, better sleep and body image, higher self-esteem, less negative and more positive mood states, and better memory and concentration (i.e. psychologial QoL). They also experience less pain and discomfort, less mobility problems and fewer limitations in daily life activities (i.e. physical QoL). Next to this, people with AUD who walk and exercise report greater participation in and opportunities for recreation / leisure and perceive their physical environment as more safe and enjoyable (i.e. environmental QoL). Lastly, those who walk more also experience more satisfying social relations and social support (i.e. social QoL). Surprisingly, such an association was not observed with the level exercising. 
Future research should explore this in more detail. Next to this, future research should also explore the effects of walking and exercising on the QoL of people with AUD. Although a previous meta-analysis demonstrated that exercise significantly reduces depressive symptoms $(\mathrm{SMD}=-0.867, \mathrm{p}=0.006)$, and improves physical fitness $(\mathrm{SMD}=0.564, \mathrm{p}=0.01)^{11}$, studies exploring the effect of exercise interventions on QoL in people with AUD are currently lacking.

In contrast with previous studies, there are two possible reasons why we did not find any associations between WHOQoL-BREF and AUDIT scores. First, previous studies ${ }^{8,20}$ were based on general population samples, while we included only institutionalized people with AUD. This explanation relates to the severity and homogeneity of alcohol use seen in this cohort. The mean AUDIT score was $18.1(\mathrm{SD}=8.4)$ indicating that the vast majority of participants were suffering serious harm as a consequence of ongoing heavy alcohol use. Statistically significant associations between QoL and alcohol consumption, as measured by AUDIT, are more likely to be found within a broader spectrum of drinkers, as seen in general population studies. Second, we did not include women in our study as female patients were not treated at the time of testing in the inpatient alcohol unit. Previous research has demonstrated that AUDIT scores in men are significantly higher than in women ${ }^{8,20}$. The current data are therefore less heterogeneous making it more complex to find any significant associations.

\section{Limitations}

First, caution should be exercised in generalising from the present findings given the limited sample size $(n=50)$ and the cross-sectional nature of this research. It was not possible to determine the direction of the association between QoL and physical activity levels in those with AUD. Longitudinal and intervention studies should be designed to identify the causal pathways between the different constructs. A second limitation is that we did not have access to hospital records of medical co-morbidity, alcohol withdrawal, or medication treatment. Therefore, the current study could not establish any relationship between QoL and these parameters. While medication could improve the general well-being it might be as well that side-effects would reduce the QoL. In the same way, we did not include data on other associated DSM 5 diagnoses, duration of (untreated) AUD, and psychiatric symptoms. It is for example known that poor QoL is associated with an earlier age of onset of alcohol use and AUDs ${ }^{21}$. Third, the majority of the included participants were hospitalised at the time of the study. Previous research demonstrated that environmental variables are significantly associated with multiple types of physical activity participation in people with mental health problems ${ }^{22,23}$. Fourth, physical activity and sedentary behaviour were measured with a self-report questionnaire which is less accurate than objective assessments ${ }^{24,25}$, as it may overestimate physical activity and underestimate sedentary levels ${ }^{26}$. Future studies should try to incorporate objective assessments of physical activity by using, for example, pedometers or accelerometers. Fifth, the different recall periods between the questionnaires used should be considered as a limitation that might affect any associations. While the SIMPAQ assesses physical activity during the previous week, the WHOQoL-BREF assesses QoL during the previous 4 weeks. Sixth, although all participants were treated in an in-patient ward, there is still a pervasive stigma associated with talking about mental illness in Uganda $^{27}$. The self-reported AUDIT scores are most likely underestimates of the real hazardous and harmful drinking patterns. Lastly, due to the lack of control data we were not able to compare the current data with any references. Nevertheless, allowing for these caveats our study is the first to investigate associations between physical activity and QoL levels in people with AUD.

\section{Conclusion}

The current findings suggest that higher levels of walking and exercising are associated with a better QoL. Our study therefore provides a platform for future research to investigate the role of physical activity on QoL levels in people with AUD in both well and low resourced settings.

\section{Acknowledgements}

None.

\section{Conflicts of interest}

The authors declare that there is no conflict of interest to report.

\section{Funding}

This project was funded by Geestkracht VZW.

\section{Role of funding source}

The funding organization had no role in the research at any stage, nor influenced the decision to publish the article. 


\section{References}

1. Connor JP, Haber PS, Hall WD. Alcohol use disorders. The Lancet. 2016;387(10022):988-98. doi: 10.1016/ S0140-6736(15)00122-1.

2. WHO. Global status report on alcohol and health 2014: World Health Organization; 2014.

3. Roerecke M, Rehm J. Cause-specific mortality risk in alcohol use disorder treatment patients: a systematic review and meta-analysis. International Journal of Epidemiology. 2014: 43(3):906-19. doi: 10.1093/ije/dyu018.

4. Reid SC, Teesson M, Sannibale C, Matsuda M, Haber PS. The efficacy of compliance therapy in pharmacotherapy for alcohol dependence: a randomized controlled trial. Journal of Studies on Alcohol. 2005;66(6):83341.

5. Foster J, Powell J, Marshall E, Peters T. Quality of life in alcohol-dependent subjects-a review. Quality of Life Research. 1999;8(3):255-61.

6. Balhara YPS, Singh S, Modak T, Sarkar S. A cross-sectional study to assess disability and its correlates among treatment seeking individuals with alcohol use disorders. Indian Journal of Psychological Medicine. 2017;39(1):40. 7. Kirouac M, Stein ER, Pearson MR, Witkiewitz K. Viability of the World Health Organization quality of life measure to assess changes in quality of life following treatment for alcohol use disorder. Quality of Life Research. 2017:1-11.

8. Kim K, Kim J-S. The association between alcohol consumption patterns and health-related quality of life in a nationally representative sample of South Korean adults. PloS One. 2015;10(3):e0119245. doi: 10.1371/ journal.pone.0119245.

9. Picci RL, Oliva F, Zuffranieri M, Vizzuso P, Ostacoli L, Sodano AJ, et al. Quality of life, alcohol detoxification and relapse: Is quality of life a predictor of relapse or only a secondary outcome measure? Quality of Life Research. 2014;23(10):2757-67.

10. Giesen ES, Deimel H, Bloch W. Clinical exercise interventions in alcohol use disorders: a systematic review. Journal of Substance Abuse Treatment. 2015;52:19. oi: 10.1016/j.jsat.2014.12.001.

11. Hallgren M, Vancampfort D, Giesen ES, Lundin A, Stubbs B. Exercise as treatment for alcohol use disorders: systematic review and meta-analysis. British Journal of Sports Medicine. 2017: 51(14):1058-1064. doi: 10.1136/bjsports-2016-096814.

12. Vancampfort D, Hert MD, Stubbs B, Soundy A, Herdt AD, Detraux J, et al. A systematic review of physical activity correlates in alcohol use disorders. Archives of Psychiatric Nursing. 2015;29(4):196-201. doi: 10.1016/j.apnu.2014.08.006.
13. Hallgren M, Vancampfort D, Schuch F, Lundin A, Stubbs B. More reasons to move: Exercise in the treatment of alcohol use disorders. Frontiers in Psychiatry. 2017. 8:160. doi: 10.3389/fpsyt.2017.00160.

14. Tremblay MS, Aubert S, Barnes JD, Saunders TJ, Carson V, Latimer-Cheung AE, et al. Sedentary Behavior Research Network (SBRN)-Terminology Consensus Project process and outcome. International Journal of Behavioral Nutrition and Physical Activity. 2017;14(1):75. doi: 10.1186/s12966-017-0525-8.

15. Biswas A, Oh PI, Faulkner GE, Bajaj RR, Silver MA, Mitchell MS, et al. Sedentary time and its association with risk for disease incidence, mortality, and hospitalization in adults: a systematic review and meta-analysis. Annals of Internal Medicine. 2015;162(2):123-32. doi: 10.7326/M14-1651

16. American Psychiatric Association. Diagnostic and Statistical Manual of Mental Disorders (DSM-5). American Psychiatric Association, Washington, DC2013.

17. World Health Organization. The world health organization quality of life (WHOQOL)-BREF. 2004 Geneva, Switzerland: World Health Organization.

18. Rosenbaum S, Ward PB. The Simple Physical Activity Questionnaire. The Lancet Psychiatry. 2016;3(1):e1. doi: 10.1016/S2215-0366(15)00496-4.

19. Babor TF, Higgins-Biddle J, Saunders J, Monteiro M. The Alcohol Use Disorders Identification TestGuidelines for use in. Primary Care (2nd Ed) Geneva, Switzerland: World Health Organization. 2001.

20. Mathiesen E, Nome S, Eisemann M, Richter J. Drinking patterns, psychological distress and quality of life in a Norwegian general population-based sample. Quality of Life Research. 2012;21(9):1527-36.

21. Fischer JA, Najman JM, Plotnikova M, Clavarino AM. Quality of life, age of onset of alcohol use and alcohol use disorders in adolescence and young adulthood: Findings from an Australian birth cohort. Drug and Alcohol Review. 2015;34(4):388-96.

22. Vancampfort D, De Hert M, De Herdt A, Bosch KV, Soundy A, Bernard PP, et al. Associations between physical activity and the built environment in patients with schizophrenia: a multi-centre study. General Hospital Psychiatry. 2013;35(6):653-8. doi: 10.1016/j.genhosppsych.2013.07.004.

23. Vancampfort D, De Hert M, De Herdt A, Soundy A, Stubbs B, Bernard P, et al. Associations between perceived neighbourhood environmental attributes and self-reported sitting time in patients with schizophrenia: A pilot study. Psychiatry Research. 2014;215(1):33-8. doi: 10.1016/j.psychres.2013.11.011

24. Soundy A, Roskell C, Stubbs B, Vancampfort D. 
Selection, use and psychometric properties of physi- Schizophr Res. 2016;176(2-3):431-440. doi: 10.1016/j. cal activity measures to assess individuals with severe mental illness: a narrative synthesis. Archives of Psychiatric Nursing. 2014;28(2):135-51. doi: 10.1016/j. apnu.2013.12.002.

schres.2016.05.017

26. Ainsworth BE, Macera CA, Jones DA, Reis JP, Addy CL, Bowles HR, et al. Comparison of the 2001 BRFSS and the IPAQ Physical Activity Questionnaires. Medicine 25. Stubbs B, Firth J, Berry A, Schuch FB, Rosenbaum S, Gaughran F, et al. How much physical activity do people with schizophrenia engage in? A systematic review, comparative meta-analysis and meta-regression. and Science in Sports and Exercise. 2006;38(9):1584-92.

27. Molodynski A, Cusack C, Nixon J. Mental healthcare in Uganda: desperate challenges but real opportunities. BJPsych International. 2017;14(4):98-100. doi:10.1192/ s2056474000002129 